\title{
BMJ Open The PATCH trial: efficacy and safety of 5\% lidocaine-medicated plaster for the treatment of patients with trigeminal neuralgia: a study protocol for a multicentric, double-blind, enriched enrolment randomised withdrawal, vehicle-controlled study
}

\author{
Chunmei Zhao (D , , ${ }^{1,2}$ Niti Shrestha (D) , ${ }^{1}$ Hongbing Liu, ${ }^{1}$ Ying Shen, ${ }^{1}$ Lan Meng, \\ Bifa Fan, ${ }^{3}$ Fang Luo (i) ${ }^{1}$
}

To cite: Zhao C, Shrestha N, Liu $\mathrm{H}$, et al. The PATCH trial: efficacy and safety of $5 \%$ lidocaine-medicated plaster for the treatment of patients with trigeminal neuralgia: a study protocol for a multicentric, doubleblind, enriched enrolment randomised withdrawal, vehiclecontrolled study. BMJ Open 2021;11:e045493. doi:10.1136/ bmjopen-2020-045493

- Prepublication history for this paper is available online. To view these files, please visit the journal online (http://dx.doi org/10.1136/bmjopen-2020 045493).

Received 02 October 2020 Accepted 13 July 2021

Check for updates

(c) Author(s) (or their employer(s)) 2021. Re-use permitted under CC BY-NC. No commercial re-use. See rights and permissions. Published by BMJ.

For numbered affiliations see end of article.

Correspondence to

Dr Fang Luo;

13611326978@163.com and

Dr Bifa Fan;

fbf1616@yeah.net

\section{ABSTRACT}

Introduction Trigeminal neuralgia (TN) is characterised by a sudden, severe, electric shock like paroxysmal pain, which is almost always associated with triggers. Carbamazepine is the first-line medical management of TN. However, side effects are common. Currently, there is no ideal treatment for TN. Since there is a known abnormality of $\mathrm{Na}^{+}$channels in the trigger zone, $5 \%$ lidocaine-medicated plaster (LMP), which can block the $\mathrm{Na}^{+}$channels on $\mathrm{A} \delta$ and $\mathrm{C}$ fibres, is an effective treatment method in many chronic pain conditions. A case report has found the benefit of LMP for the treatment of TN without any side effects. Whether LMP is an option for the treatment of TN is worth exploring.

Methods and analysis The PATCH trial is a double-blind, enriched enrolment with randomised withdrawal, vehiclecontrolled trial, aiming to explore the effects and safety of LMP in patients with TN. There is a 3-week initial openlabel phase, followed by a 4-week double-blind treatment phase for responders. In the double-blind phase, patients will have to withdraw from this PATCH study if they meet one of the following criteria for treatment failure such as: $>50 \%$ increase in pain intensity or paroxysms, lack of efficacy or side effects. The primary outcome will be the number of treatment failures. Adverse events will also be monitored throughout the study.

Ethics and dissemination This study protocol has been approved by the Institutional Review Board of Beijing Tiantan Hospital (approval number: KY 2020-102-02). The results will be disseminated in international academic meetings and published in peer-reviewed journals. Trial registration number NCT04570293.

\section{INTRODUCTION}

Trigeminal neuralgia (TN) is characterised by a sudden, severe, usually unilateral, transient, stinging, recurrent electric shock like pain in one or more divisions of the trigeminal
Strengths and limitations of this study

- Considering the high failure rates of analgesic drugs, this PATCH trial uses the enriched enrolment with randomised withdrawal (EERW) design including a 3-week initial open-label phase, followed by a 4week double-blind treatment phase for responders.

- This multicentric, double-blind, EERW, vehiclecontrolled trial aims to establish a high-quality evidence in the utility of lidocaine-medicated plaster for trigeminal neuralgia.

- Patients will be participating in a double-blind treatment period of 4 weeks and the optimal duration of treatment remains to be studied in the future.

nerve, lasting from a few seconds to less than 2 min. ${ }^{1}$ Onset is usually after 40 years of age, with a peak between 50 and 60 years of age. Simple daily-life activities, such as washing face, brushing teeth, eating and drinking or a slight touch of trigger points may prompt the attack of TN, ${ }^{2}$ resulting in a decline in the patient's quality of life (QoL). Trigger zones are predominantly located in the perioral and nasal regions. Paroxysmal pain is associated with triggers in virtually all patients with TN.

Rappaport and Devor proposed the ignition hypothesis of $\mathrm{TN}$ on pain paroxysms and mechanical compression of the trigeminal root. ${ }^{3}$ Sensory axon demyelination in the trigeminal root due to sustained (static) or pulsatile microvascular compression of the trigeminal root has been proposed as the primary pathogenic factor. ${ }^{45}$ There are abnormalities of receptors or nerve endings in the trigger zone, such as the abnormality 
of $\mathrm{Na}^{+}$channels. ${ }^{6}$ Even minimal stimulations, such as light touch, talking or chewing, may cause all $\mathrm{Na}^{+}$channels to open. Pain bouts begin with impulses in a small set of injured axons that have been rendered hyperexcitable from trigger point stimulation. These impulses are conducted antidromically in peripheral afferents, and neighbouring fibres are excited through synchronisation after discharge activity. Activity then spreads beyond the original focal fibre discharge, and pain occurs. Based on this hypothesis, TN may be caused by the abnormality of the trigger zone; hence, the blockade of $\mathrm{Na}+$ channels of the trigger zone may be a novel and effective treatment method for $\mathrm{TN}^{7}$

Systemic analgesics are regularly employed as part of a treatment regimen of $\mathrm{TN}^{8}$ Carbamazepine (oxcarbazepine for intolerant individuals) is recommended as the first-line of treatment for TN by blocking voltage gated $\mathrm{Na}^{+}$channels; however, carbamazepine has many side effects. The most common side effects are dizziness, drowsiness, hyponatraemia, nausea and vomiting, blood disorders and hypersensitivity reactions (eg, rashes) ${ }^{9} \mathrm{~A}$ multitude of complications have been reported as reasons for carbamazepine discontinuation. The second-line of treatments for $\mathrm{TN}$ including lamotrigine, baclofen and pimozide, are based on very little evidence ${ }^{10-12}$ Currently, due to these increased tolerability problems and drug withdrawals, especially in long-term use, clinicians and patients are seeking more feasible options for TN pain relief.

The use of topical agents is one of the most basic approaches in the pharmacological management of neuropathic pain. For example, creams, gels, foam sprays and solutions containing lidocaine are commonly used for short-term analgesia. ${ }^{13}$ Lidocaine is usually applied as a plaster to treat chronic pain. ${ }^{14-16} 5 \%$ lidocainemedicated plaster (LMP) is a white hydrogel plaster containing adhesive material. Psychophysical data indicates that $5 \%$ lidocaine produces partial sensory block of $\mathrm{Na}^{+}$channels on $\mathrm{A} \delta$ and $\mathrm{C}$ fibres, with no change in large myelinated $\mathrm{A} \beta$ sensory fibre function. ${ }^{17}$ This blockade reduces ectopic discharge and signals transduction at the site of application. Furthermore, LMP acts on the peripheral mechanism of pain pathway and has been found to be well-tolerated and non-addictive with minimal risks for unwanted drug-drug interactions and systemic adverse events (AEs)..$^{18} 19$

In 1999, LMP was approved for the treatment of postherpetic neuralgia (PHN) by the US Food and Drug Administration.$^{2021}$ In addition to being the first-line treatment for PHN, LMP also seems to be an effective treatment method for osteoarthritis pain, ${ }^{22}$ postsurgical and posttraumatic pain, ${ }^{23}$ nerve injury pain ${ }^{24}$ and painful peripheral neuropathy. ${ }^{25-27}$ Tamburin $e t a l^{28}$ first reported that wo patients with primary $\mathrm{TN}$ were instructed to wear LMP over the affected area after stopping the intake of oral medications because of side effects or refusal to undergo surgical procedures; their results show significant reduction in intensity and number of pain paroxysms without any side effects. However, the number of cases was small and the observed reduction in pain intensity may have been due to treatment effect, placebo effect, changes in underlying disease state or a combination of these factors. Therefore, whether LMP is a good option for the treatment of TN is worth exploring.

However, high failure rates of analgesic drugs, particularly in chronic pain conditions, should also be considered. ${ }^{29}$ Therefore, we have designed this double-blind, enriched enrolment with randomised withdrawal (EERW), vehicle-controlled trial, in which we aim to explore the effects and safety of LMP in patients with TN. We hypothesise that by blocking $\mathrm{Na}^{+}$channels of facial trigger zone and pain area, LMP may provide a novel, valuable, localised and targeted approach for treating TN.

\section{METHODS AND ANALYSIS}

The EERW design specifically focuses on chronic conditions, such as chronic pain. ${ }^{30}$ Recently, there have been several literatures successfully applying the EERW design method to study chronic pain conditions. ${ }^{151-33}$ Conventional clinical trials report the average response of treatment. Whereas, a meta-analysis reveals that some patients have good response, others have limited benefits and a few have average effect. ${ }^{34}$ Therefore, the actual effect within subgroups may be masked by the average effect. Significant difference between the EERW design and the classic randomised controlled trial design is the timing of entry of participants into randomisation. Participants in the EERW design undergo an initial process of active drug titration prior to randomisation, and only the participants who achieve satisfactory results without intolerable AEs are eligible for subsequent randomisation. This open drug titration process will be performed in routine clinical practice and will provide data on the proportion of responders and non-responders to drug therapy, the optimal dose of the analgesic drug and the proportion of withdrawal due to AEs. In addition, during the traditional placebo-controlled studies, many participants will be exposed to placebo. However, because of the natural course of pain, there are many controversial medical and ethical dilemmas on directly subjecting participants to either placebo treatment or no treatment. In the EERW design, only participants who achieve satisfactory effect after being openly titrated are allowed to receive subsequent placebo treatment, thereby greatly reducing the proportion of subjects receiving placebo treatment.

This PATCH trial has been approved by the Institutional Review Board of Beijing Tiantan Hospital (approval number: KY 2020-102-02). This will be a prospective, double-blinded, vehicle-controlled, parallel-group, multicentre, EERW trial aimed at estimating the efficacy and safety of LMP in patients with TN, with a 1:1 allocation ratio during the double-blind randomisation phase (see figure 1). A total of 310 patients will be enrolled within a 3-year inclusion period. Online supplemental file 1 shows 


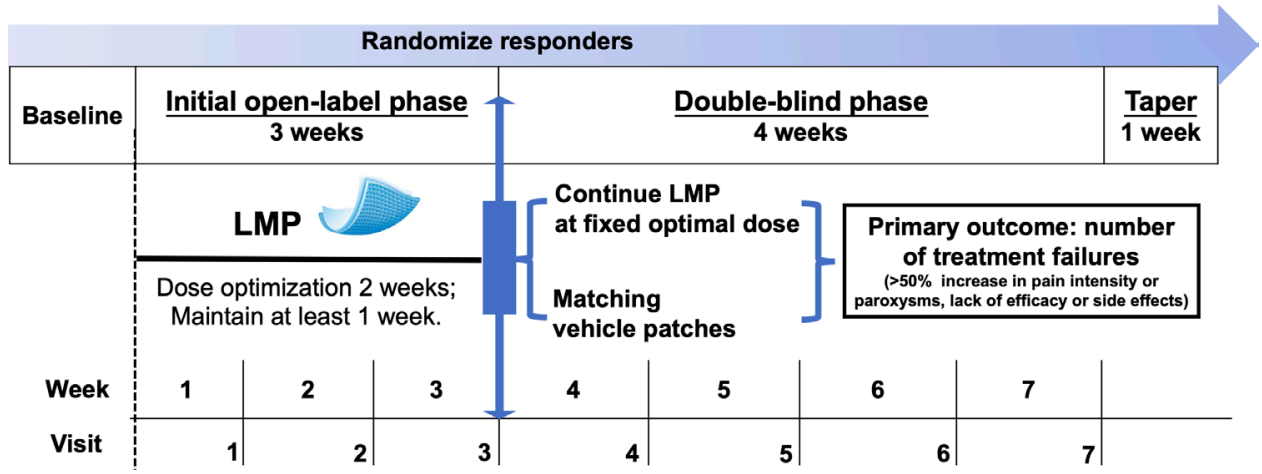

Figure 1 Flow chart of the study procedure. LMP, 5\% lidocaine-medicated plaster.

the Standard Protocol Items: Recommendations for Interventional Trials (SPIRIT) checklist for study protocols.

\section{Study design}

\section{Baseline assessment}

Baseline registration will be performed before treatment. At baseline assessment, participants will provide the demographic data and a review of history of $\mathrm{TN}$, including age at onset, side, anatomical localisation of pain, intensity of pain, neuropathic symptoms, duration of disease, number and duration of paroxysms, concomitant continuous pain and pain remission periods. History of prior treatment, history of medication and concomitant medications will also be collected using a dedicated questionnaire. Baseline pain assessment will include the use of Brief Pain Inventory-Short Form (BPI-SF) ${ }^{35}$ and the recording of global assessments to measure patient response to existing (prestudy) medications. Subsequently, the 3-week initial open-label treatment phase will be initiated.

\section{Initial open-label treatment phase}

After providing informed consent and completing a baseline evaluation, patients will participate in an initial openlabel treatment period of 3 weeks of LMP (active patches) containing $700 \mathrm{mg}$ lidocaine $(5 \% \mathrm{w} / \mathrm{w}$ ) (Versatis, Tide Pharmaceutical, Beijing, China), measuring $10 \times 14 \mathrm{~cm}$. The investigators will cut the lidocaine patches into four pieces, $7 \times 5 \mathrm{~cm}$ each. Cutting the patch is an appropriate use of the product, according to the manufacturer's prescribing information, and will not interfere with drug delivery from the patch because distribution of the drug is homogeneous throughout the patch. For each patient, painful area and trigger point will be chosen for treatment. The patches will be applied to the skin overlying the affected areas for 12 hours/day. The patches can be applied during the night (application in the evening and removal in the morning), or during the day. Patients will be told to apply up to three patches at one time and will be permitted to titrate down to a lower dose. Patients will be required to maintain the optimal dose for at least 1 week. Patients will be instructed to maintain a diary of response to the LMP and record any untoward reactions experienced during the 3-week initial open-label treatment period.

A responder at the end of the open-label treatment phase will be categorised as follows:

- A 30\% or more decrease in mean daily pain intensity for the 7 days prior to the 21 st day, compared with that in the baseline phase when the LMP is applied and pain returns or increases when LMP is removed.

- A $30 \%$ or more decrease in the total number of paroxysms for the 7 days prior to the 21st day, compared with that in the baseline phase.

- Regular plaster use will be defined as the plaster applied every 2 days.

- No intolerable side effects occur at the dose of the existing patch.

The responders will be included in the subsequent double-blind treatment phase and patients who do not meet the inclusion criteria for double-blind treatment phase will cease participation following a 1-week drug taper phase.

\section{Subsequently double-blind treatment phase Randomisation}

The SPSS V.25.0 software (IBM) will be used to conduct a random allocation sequence in a 1:1 ratio by the Clinical Research Coordinator (CRC) for this trial. An independent CRC will be responsible for randomisation, enrolment and assigning of participants to different groups. Randomisation will be stratified by centre. Block randomisation in a 1:1 ratio will be used within each stratum (ie, centre). Randomly allocated information will be inserted into sealed, opaque envelopes that will be kept in a double locked cabinet, and sent to each centre. Group allocation will be concealed until the final data analysis is completed. Random allocation will be performed on day 21. A total of 310 patients will be initially recruited in order to ensure random equal allocation of 124 responders to continue LMP (LMP group) or receive vehicle patches (control group).

\section{Blinding}

Participants, physicians, nurses, outcome assessors and data analysts will be blinded to treatment allocation. To ensure blinding, the vehicle patches will be identical to the 
active patches in all aspects such as packaging, colour and size, except for the absence of lidocaine. After obtaining treatment number from the CRC, the pharmacologists involved in this study will prepare corresponding patches and attach treatment number labels to participant's case report form (CRF).

\section{Treatment during the double-blind treatment phase}

In the double-blind treatment phase, eligible patients will be randomly assigned to continue LMP at optimal dose achieved in the flexible-dose open-label treatment phase (LMP group) or to receive vehicle patches (control group). The dose of LMP achieved at day 21 will remain unchanged for 4 weeks during the subsequent doubleblind period in the LMP group. For patients in the control group, a strict vehicle patches regimen will be followed for the next 4 weeks. Regardless of allocation, no further titration will be performed in the double-blind treatment phase. After the end of the double-blind treatment phase or premature discontinuation, a 1-week taper phase will be incorporated to reduce the risk of potential withdrawal effects for patients assigned to the LMP group. Similarly, patients in the control group will also taper their vehicle patches after the end of the doubleblind treatment phase or premature discontinuation.

\section{Additional treatment}

During the treatment period, patients will maintain on their current systemic analgesic regimen from the screening visit, with no dose adjustment or additions allowed other than the LMP or vehicle patches. The patches will be added on top of the existing analgesic treatment. Patients will be allowed to use over-the-counter medications such as acetaminophen, acetylsalicylic acid or other non-steroidal anti-inflammatory drugs (NSAIDs) for pain not related to this study (eg, headache or fever). At the end of this study, all patients will be allowed to request administration, or additional administration of the standard analgesic treatment regimen if previous pain relief is unsatisfactory.

\section{Participants}

Participant recruitment

Besides the lead centre at Beijing Tiantan Hospital in Beijing, participants will also be recruited from other 17 hospitals within China. Eligible subjects will be given an explanation of the study by the CRC, and will voluntarily sign a consent form consenting their participation.

\section{Inclusion criteria}

Patients aged $18-80$ years who provide a written informed consent will be eligible to participate in this PATCH study, if they have a diagnosis of TN based on the third edition of the International Classification of Headache Disorders criteria. ${ }^{1}$ The diagnosis of TN will be confirmed by two pain physicians. The inclusion criteria are:

- Occurrence of episodes of intense facial paroxysmal pain in the distribution(s) of one or more divisions of the trigeminal nerve including exclusive to V1, triggered by innocuous stimuli.

- Average daily pain intensity $\geq 4$ by a BPI-SF item 5 score ( $0-10$ rating scale of average pain) in the preceding 24-hour period.

- Concomitant analgesic regimens that include 14 days of stable doses with systemic analgesics rather than topical agents for the relief of PHN will be permitted.

- Normal neurological examination.

- Normal neuroimaging analysis.

\section{Exclusion criteria}

- Atypical pain location (eg, no specific trigger points) or trigger zones in the mouth.

- Proposed surgical intervention due to preference of patient.

- Any condition known to interfere with the correct execution of sensory tests (eg, peripheral or central neurological dysfunction or cognitive impairments).

- Presence of any other acute or chronic pain disorder with the need of systemic analgesic medication for more than 10 days in the last 3 months.

- Inability to discontinue the use of another lidocainecontaining products or a class I antiarrhythmic drug during the study period.

- History of hypersensitivity to an amide-type local anaesthetic agent, or other contents of the lidocaine or vehicle patch.

- History of surgical intervention or neurological ablation to treat TN.

- Participation in another clinical trial within 30 days of the study.

- Any patient who was judged to be unreliable or unable to understand the study protocol.

- Any abnormality of the skin or of vascular origin at application site.

- Pregnancy or breast feeding.

\section{Subject withdrawal criteria}

Participants will be informed that they are permitted to withdraw from the PATCH study voluntarily at any stage of the trial, for any reason.

\section{Efficacy outcomes}

\section{Primary outcome}

The primary outcome will be the number of treatment failures on LMP versus the number of treatment failures on vehicle patches throughout the double-blind treatment phase. Patients will be categorised as treatment failure if one of the following situations occur:

- A 50\% or more increase in mean daily pain intensity experienced in the paroxysms within the 7-day period of double-blind treatment phase compared with that at the end of the initial open-label treatment phase.

- A $50 \%$ or more increase in the total number of paroxysms within the 7-day period of double-blind treatment phase, compared with that at the end of the initial open-label treatment phase. 
- Patient discontinues intervention due to lack of efficacy or intolerable side effects associated with the study patches.

On every seventh day of follow-up in the double-blind phase, patients will be assessed for treatment failure. Patients meeting the criteria for treatment failure will be withdrawn from this study.

\section{Secondary outcomes}

- Time to loss of therapeutic response (LTR) will be defined as the number of days to treatment failure in the double-blind phase after randomisation.

- Proportion of responders and non-responders at the end of open-label period.

- Pain intensity of items 3-6 on the BPI-SF will be recorded daily by the patients. Patients will be asked to circle the number on an 11-point Likert scale of 0 (no pain) to 10 (worst pain imaginable) that describes their worst pain (worst pain), their least pain (least pain) and their pain on average (average pain) in the last 24 hours and how much pain they are experiencing at the time of the evaluation (pain right now).

- The number and severity of paroxysms experienced in the past 4 days will also be recorded weekly.

- Pain relief will be assessed by item 8 on the BPI-SF. Patients will be asked to circle the percentage value from $0 \%$ (no relief) to $100 \%$ (complete relief) that shows how much pain relief they have achieved during the last 24 hours. The proportion of patients who report pain relief of $50 \%$ or greater at the end of the open-label phase, and at the end of double-blind phase or premature discontinuation from baseline will be recorded.

- The number-needed-to-treat (NNT) is an estimate of how many patients must receive active treatment to achieve response in one patient. The NNT of this study will be defined as the number of enrolled patients with $\mathrm{TN}$ to obtain one patient with $>50 \%$ pain relief with LMP.

- Pain interference with the QOL will be assessed by items 9A-G on the BPI-SF. On these items, patients will be asked to circle the number on an 11-point Likert scale of 0 (does not interfere) to 10 (completely interferes), which describes the extent to which pain has interfered with their activities of daily living during the last 24 hours. The seven items are: general activity; mood; walking ability; normal work; relations with other people; sleep; and enjoyment of life. The items 9A-G will be examined individually on a weekly basis.

- Pain interference with sleep will be assessed by the Pittsburgh Sleep Quality Index (PSQI) ${ }^{36}$ at baseline, at the end of open-label phase, and the end of doubleblind phase or premature discontinuation. Nineteen individual items generate seven 'component' scores: subjective sleep quality, sleep latency, sleep duration, habitual sleep efficiency, sleep disturbances, use of sleeping medication and daytime dysfunction. The sum of scores for these seven components yield one global score. The total score is 21. The higher the scores, the worse the sleep.

- The patients' health situation will be assessed by the short form 36-Item Short Form Survey (SF-36) health survey questionnaire ${ }^{37}$ at baseline, at the end of open-label phase, and at the end of double-blind phase or premature discontinuation. It measures health on eight multi-item dimensions, covering functional status, well-being and an overall evaluation of health.

- At the end of open-label phase, and at the end of double-blind phase or premature discontinuation, the patients' overall health status will be assessed by Patient Global Impression of Change (PGIC) (a 7-point Likert scale, where $1=$ very much improved to $7=$ very much worse with a value of 4 representing no change).

- The cost of treatment will include the total cost of all medications for TN along with the cost of LMP.

- Evaluation of study blinding: Both the clinician and the patient will guess that the drug used during double-blind phase is LMP or vehicle patches.

\section{Monitoring of all types of AEs}

Safety and tolerability will be assessed via AEs monitoring. Patients will be asked to record any AEs of the therapy throughout the study period. Even if patients are unsure whether the change is related to the treatment they are receiving, they will be asked to note any unusual (generalised or local) change in their health status (apart from their TN-related pain). The date and time of occurrence, development of $\mathrm{AE}$ and the duration of $\mathrm{AE}$ will be recorded and any $\mathrm{AE}$ will be immediately reported to the study investigator.

Erythema, oedema and the presence of papules or vesicles will be assessed using dermal evaluations. The degree of dermal irritation at each application site will be assessed by using a 5 -point scale $(0=$ none to $4=$ severe $)$. Each application site will be tested for sensation using light touch and pinprick at baseline (prior to application of the patch), and then at the end of open-label phase and at the end of double-blind phase or premature discontinuation. For light touch, each patient will receive a single stroke with a cotton swab over the area of patch application. For the pinprick test, a single pinprick will be made in the area of patch application. Application sites will be scored as either a positive $(+)$ or a negative $(-)$ sensation to light touch or pinprick.

\section{Follow-ups}

Patients will be contacted via telephone by a study nurse, weekly. At the end of open-label phase, and at the end of double-blind phase or premature discontinuation, patients will be scheduled to return to the clinic for a detailed evaluation by the treating physician. The detailed evaluation will consist of complete assessments (BPI-SF, PSQI, SF-36 and PGIC), a skin evaluation and the recording of AEs, if any. 


\section{Sample size calculation}

A sample size of 124 responders in the double-blind phase (62 patients per group) will provide $90 \%$ power to detect a difference of the primary outcome based on an $\alpha$ of 0.05 (two sided), assuming a $30 \%$ failure rate for LMP and a $58 \%$ failure rate for vehicle patches. The estimated effect value mainly refers to the data from a previous EERW study of LMP. ${ }^{15}$ To our knowledge, only one case report has reported the efficacy of LMP in TN, in which, all patients achieved satisfactory pain relief. ${ }^{28}$ Another retrospective observational study demonstrated that $53 \%$ patients with localised neuropathic pain conditions responded poorly to pharmacological therapy, following the use of LMP obtained pain relief of $50 \%$ or $^{2}$ reater. ${ }^{38}$ We conservatively hypothesise that about $50 \%$ patients in the initial open-label phase will achieve satisfactory pain relief. A total of 248 patients entering the open-label phase will be initially estimated to provide the required 124 responders. In addition, considering a drop-out rate of $20 \%$, we have decided to recruit 310 patients.

\section{Statistical analyses \\ Baseline characteristics}

All statistical analyses of the data will be performed by using SPSS software V.25.0 (SPSS). Patient characteristics and descriptive variables will be presented for each treatment arm: sex, age, side, intensity, duration and triggers sites of pain, duration of medical treatment prior to study (days) and prestudy global assessments (BPI-SF, PSQI and SF-36). The normal distribution of continuous variables will be tested by the Kolmogorov-Smirnov test. Continuous variables will be presented as means and SD and comparisons using the independent t-test will be performed if they are normally distributed. Continuous variables will be presented as medians with IQRs compared using the Wilcoxon's rank-sum test if they are not normally distributed. Categorical variables will be presented as frequencies and percentages and compared using $\chi^{2}$ or Fisher's exact tests.

\section{Primary outcome}

Both modified intention-to-treat analysis (ITT) and perprotocol (PP) analysis will be used for primary outcome to determine robustness of evidence. For modified ITT analysis, all patients who are randomised into the double-blind phase of the study and receive at least one of the study patches will be analysed. For PP analysis, participants who drop-out of the study will be excluded from analysis. For the follow-up outcomes at the end of this study, the proportion of patients with follow-up and who are lost to follow-up will be presented for each treatment arm. Primary outcome, number of treatment failures, will be described as the difference between frequencies and percentages between the two groups. $\chi^{2}$ or Fisher's exact tests will be used to detect the difference between the two groups. The level for declaring significant difference will be a two-sided $\mathrm{p}$ value $(\mathrm{p}<0.05)$. No interim analysis has been planned. Subgroup analyses will also be conducted to explore any potential differences in outcomes by study centre, age, sex, daily pain score (baseline), site of trigger and history of oral pain medication. Breslow-Day test will be used to evaluate the differences among the centres. If there are centre effects, logistic regression analysis will be performed, and the centre will be adjusted as a covariate to analyse the results of interventions and clinical assessment.

\section{Secondary outcomes}

Patients who meet treatment failure will be withdrawn from the study; evaluations after withdrawal will be excluded from statistical analysis. We will plot LTR using Kaplan-Meier curves, and compare treatment groups using the log-rank test. For secondary outcomes that are continuous variables (eg, items $9 \mathrm{~A}-\mathrm{G}$ on the BPISF, PSQI scores and SF-36 scores, PGIC, cost of treatment), the difference between the two means with $95 \%$ CIs will be presented. Independent t-test or Wilcoxon's rank-sum test will be used to detect the difference between the two groups. Dichotomous outcomes (eg, proportion of responders and non-responders) will be described as the absolute number and proportion. A $\chi^{2}$ or Fisher's exact test will be used. These analyses will be viewed as hypothesis generating; therefore, corrections for multiple testing have not been planned. For weekly mean pain scores of items 3-6 on the BPI-SF and the number of paroxysms, the repeated measures analysis of variance will be performed with treatment as the between-subject factor, and the visit and its interaction with treatment as the within-subject factors. All other continuous variables will be analysed using analysis of covariance, controlling for baseline values. Missing observations will be replaced with the prior observation carried forward.

\section{Safety analysis}

All patients who receive one or more patches of LMP will be included in the safety analysis. All safety data will be compiled and included in the final report. Incidence of AEs will be summarised and presented as a form.

\section{Data management and monitoring}

Data will be collected in a CRF by dedicated clinical trial assistants. All documents related to the trial will be recorded and labelled with participant identification codes. Participants' identification data will be kept confidential and will not show the name of the participant until the results of the study is published. Raw data (CRFs) and written informed consent will be stored in a double-locked cabinet.

When the investigators enter the records in CRF, they will be required to ensure the accuracy of the data. Data monitoring and validation will be independently reviewed by the data and safety monitoring committee (DSMC). On the basis of monitoring, the DSMC may consider early termination of this treatment regimen, if treatment-related serious AEs were to occur during enrolment. Meanwhile, protocol compliance, safety and study progress will also be monitored by the DSMC. 


\section{Patient and public involvement}

Patients and the public will not be involved in any of the phases of this study.

\section{Ethics and dissemination}

We have followed the SPIRIT statement $2013^{39}$ and the Consolidated Standards of Reporting Trials guidelines. ${ }^{40}$ The study will be conducted in accordance with the Declaration of Helsinki. ${ }^{41}$ The results of the study will be communicated and disseminated at national and international conferences of pain and neurology, as well as through peer-reviewed manuscripts published in open access journals. At the completion of the trial and following publication of primary and secondary outcomes, requests for data sharing will be considered by the PATCH trial Management Group.

\section{DISCUSSION}

This multicentric, double-blind, EERW, vehicle-controlled trial aims to establish a high-quality evidence in the utility of LMP for TN. We hypothesise that LMP may provide pain relief and improve patients' QOL, with minimal risk for additional side effects or drug interactions in patients with TN. LMP may represent a promising treatment for $\mathrm{TN}$, a disease where there is a consistent mismatch between therapeutic guidelines and patient's preferences and expectations.

The EERW design approach has previously been used in several studies on chronic pain, such as TN, PHN and diabetic peripheral neuropathic pain. ${ }^{15}$ 31-33 However, there has been no consensus on time period (eg, 3+4 weeks, $8+2$ weeks, $6+14$ weeks, $4+5$ weeks). Considering that different types of chronic pain have different characteristics, we referred to a study on TN using the EERW design approach ${ }^{33}$ and defined the time period as $3+4$ weeks (a 3-week initial open-label phase followed by a 4-week double-blind treatment phase for responders).

Lidocaine is applied as a plaster, with steady release of the drug through the skin of the affected area; the amount of lidocaine that penetrates is enough to cause analgesia, but not anaesthesia. The lidocaine-induced reduction in sensory input from trigger points by LMP may prevent cutaneous triggering. The therapeutic effect of lidocaine is unlikely to be the result of systemic absorption, which has been reported to be minimal. ${ }^{42}{ }^{43}$ Additionally, long-term exposure to LMP is not expected to lead to any safety concerns. ${ }^{18}$ Prescribed systemic medications for TN may be associated with AEs such as sedation, dizziness, nausea and vomiting or liver and kidney damage. These AEs may be particularly problematic in $\mathrm{TN}$ patients. When combined with systemic medications, LMP reduces the risk of systemic toxicity of oral drugs. Currently, LMP is the first choice for treatment of PHN. Both PHN and TN are neuropathic pain conditions. However, most of the previous studies on LMP applied for treating TN were case series. ${ }^{28}$ Therefore, there is an urgency to undertake a large-scale study that provides the best evidences to determine if the use of LMP provides significant pain relief in $\mathrm{TN}$.

There are several limitations to our study. First, there are patient compliance issues. Patients may withdraw due to side effects of the drug or some other reasons in the initial open-label phase and the subsequent double-blind phase. In order to reduce withdrawal rate, we will provide thorough guidance to the patients before recruitment and ensure adherence by writing down the details of prescriptions in small notes. Second, pressure pain thresholds and electrical pain thresholds are not measured at trigger points and affected areas in this study. If the results of this study are positive, we will further perform the evaluation of somatic pain sensitivity, in which electrophysiological technology will be added. Third, LMP can only resolve facial pain in the trigeminal nerve distribution area, but cannot relieve pain in the mouth or other trigger zones in the oral mucosa. Pain or trigger zones in the mouth needs to be treated with other treatments because LMP can only be applied externally to the skin. Fourth, carry-over effects may also occur from initial open-label period to subsequently double-blind period because patients are very likely to know they are receiving placebo vs LMP during double-blind phase. Furthermore, this concept may be exaggerated by the initial open-label period-where patients learn how the patch feels and if it helps them. This phenomenon will exaggerate a positive response for the LMP over control. Fifth, we only use a single concentration of LMP and if the results are positive, a dose-effect response trial on the concentration of LMP (eg, 0.5\% LMP and 5\% LMP) and the effectiveness of pain relief needs to be performed. Finally, patients will be participating in a double-blind treatment period of 4 weeks. The optimal duration of treatment remains to be studied in the future.

Lastly, if the results of this PATCH trial turn out to be positive, this study will provide strong evidence for using LMP for the treatment of TN in future clinical practice. LMP may be used as a first line of treatment and can also be a perfect add-on therapy in a multidrug concept for patients with $\mathrm{TN}$.

\section{Trial status}

The PACTH trial will start recruiting participants in January 2021 and is on track to complete enrolment of 310 participants by December 2023.

\section{Author affiliations}

${ }^{1}$ Department of Pain Management, Beijing Tiantan Hospital, Capital Medical University, Beijing, China

${ }^{2}$ Beijing Tiantan Hospital, Beijing, China

${ }^{3}$ National Pain Management \& Research Center, China-Japan Friendship Hospital, Beijing, China

Twitter Niti Shrestha @NitiShrestha

Contributors CMZ and NS contributed equally to this work and should be considered co-first authors. CZ participated in the study design and drafted the manuscript. HL, YS and LM participated in the study design and performed critical revision of the manuscript. FL and BF are the principal investigator of the entire 
study and contributed equally to this work. All authors read and approved the final manuscript.

Funding This research was supported by the Capital's Funds for Health Improvement and Research (No. 2020-2-2046 and Beijing Municipal Administration of Hospitals Clinical Medicine Development of Special Funding Support (No. XMLX201707)

Disclaimer The funder is not involved in subject recruitment, intervention, data collection, data analysis or preparation of the manuscript.

\section{Competing interests None declared.}

Patient and public involvement Patients and/or the public were not involved in the design, or conduct, or reporting, or dissemination plans of this research.

Patient consent for publication Parental/guardian consent obtained.

Provenance and peer review Not commissioned; externally peer reviewed.

Open access This is an open access article distributed in accordance with the Creative Commons Attribution Non Commercial (CC BY-NC 4.0) license, which permits others to distribute, remix, adapt, build upon this work non-commercially, and license their derivative works on different terms, provided the original work is properly cited, appropriate credit is given, any changes made indicated, and the use is non-commercial. See: http://creativecommons.org/licenses/by-nc/4.0/.

\section{ORCID iDs}

Chunmei Zhao http://orcid.org/0000-0001-7734-4192

Niti Shrestha http://orcid.org/0000-0001-7360-9747

Fang Luo http://orcid.org/0000-0002-3584-0355

\section{REFERENCES}

1 Headache Classification Committee of the International Headache Society (IHS). The international classification of headache disorders, 3rd edn. Cephalalgia2018;38:1-211.

2 Magrinelli F, Zanette G, Tamburin S. Neuropathic pain: diagnosis and treatment. Pract Neurol 2013;13:292-307.

3 Rappaport HZ, Devor M. Trigeminal neuralgia: the role of selfsustaining discharge in the trigeminal ganglion. Pain 1994;56:127-38.

4 Devor M, Govrin-Lippmann R, Rappaport ZH. Mechanism of trigeminal neuralgia: an ultrastructural analysis of trigeminal root specimens obtained during microvascular decompression surgery. J Neurosurg 2002;96:532-43.

5 Devor M, Amir R, Rappaport ZH. Pathophysiology of trigeminal neuralgia: the ignition hypothesis. Clin J Pain 2002;18:4-13.

6 Liu M, Zhong J. Mechanism underlying cranial nerve rhizopathy. Med Hypotheses 2020;142:109801.

7 Liu J, Dai J, Lingling E, et al. Trigeminal neuralgia may be caused by abnormality of the trigger zone. Med Hypotheses 2010;74:818-9.

8 Bendtsen L, Zakrzewska JM, Heinskou TB, et al. Advances in diagnosis, classification, pathophysiology, and management of trigeminal neuralgia. Lancet Neurol 2020;19:784-96.

9 Wiffen PJ, Derry S, Moore RA, et al. Carbamazepine for chronic neuropathic pain and fibromyalgia in adults. Cochrane Database Syst Rev 2014:4:CD005451.

10 Tentolouris-Piperas V, Lee G, Reading J, et al. Adverse effects of anti-epileptics in trigeminal neuralgiform pain. Acta Neurol Scand 2018;137:566-74.

11 Fromm GH, Terrence CF, Chattha AS. Baclofen in the treatment of trigeminal neuralgia: double-blind study and long-term follow-up. Ann Neurol 1984;15:240-4.

12 Lechin F, van der Dijs B, Amat J, et al. Definite and sustained improvement with pimozide of two patients with severe trigeminal neuralgia. Some neurochemical, neurophysiological and neuroendocrinological findings. J Med 1988;19:243-56.

13 Moore D, Chong MS, Shetty A, et al. A systematic review of rescue analgesic strategies in acute exacerbations of primary trigeminal neuralgia. Br J Anaesth 2019;123:e385-96.

14 Bischoff JM, Petersen M, Uçeyler N, et al. Lidocaine patch (5\%) in treatment of persistent inguinal postherniorrhaphy pain: a randomized, double-blind, placebo-controlled, crossover trial. Anesthesiology 2013;119:1444-52.

15 Binder A, Bruxelle J, Rogers P, et al. Topical 5\% lidocaine (lignocaine) medicated plaster treatment for post-herpetic neuralgia: results of a double-blind, placebo-controlled, multinational efficacy and safety trial. Clin Drug Investig 2009;29:393-408.

16 Cheville AL, Sloan JA, Northfelt DW, et al. Use of a lidocaine patch in the management of postsurgical neuropathic pain in patients with cancer: a phase III double-blind crossover study (N01CB). Support Care Cancer 2009;17:451-60.

17 Krumova EK, Zeller M, Westermann A, et al. Lidocaine patch (5\%) produces a selective, but incomplete block of $A \delta$ and $C$ fibers. Pain 2012;153:273-80.

18 Bursi R, Piana C, Grevel J, et al. Evaluation of the population pharmacokinetic properties of lidocaine and its metabolites after long-term multiple applications of a lidocaine plaster in postherpetic neuralgia patients. Eur J Drug Metab Pharmacokinet 2017:42:801-14.

19 Gammaitoni AR, Alvarez NA, Galer BS. Safety and tolerability of the lidocaine patch $5 \%$, a targeted peripheral analgesic: a review of the literature. J Clin Pharmacol 2003;43:111-7.

20 Gammaitoni AR, Alvarez NA, Galer BS. Safety and tolerability of the lidocaine patch $5 \%$, a targeted peripheral analgesic: a review of the literature. J Clin Pharmacol 2003;43:111-7.

21 Fleming JA, O'Connor BD. Use of lidocaine patches for neuropathic pain in a comprehensive cancer centre. Pain Res Manag 2009;14:381-8.

22 Burch F, Codding C, Patel N, et al. Lidocaine patch 5\% improves pain, stiffness, and physical function in osteoarthritis pain patients. A prospective, multicenter, open-label effectiveness trial. Osteoarthritis Cartilage 2004;12:253-5.

23 Hans G, Joukes E, Verhulst J, et al. Management of neuropathic pain after surgical and non-surgical trauma with lidocaine $5 \%$ patches: study of 40 consecutive cases. Curr Med Res Opin 2009;25:2737-43.

24 Madsen CS, Johnsen B, Fuglsang-Frederiksen A, et al. Differential effects of a $5 \%$ lidocaine medicated patch in peripheral nerve injury. Muscle Nerve 2013;48:265-71.

25 Meier T, Wasner G, Faust M, et al. Efficacy of lidocaine patch 5\% in the treatment of focal peripheral neuropathic pain syndromes: a randomized, double-blind, placebo-controlled study. Pain 2003;106:151-8.

26 Meier T, Faust M, Hüppe M, et al. [Reduction of chronic pain for nonpostherpetic peripheral neuropathies after topical treatment with a lidocaine patch]. Schmerz 2004;18:172-8.

27 Rehm S, Binder A, Baron R. Post-herpetic neuralgia: 5\% lidocaine medicated plaster, pregabalin, or a combination of both? A randomized, open, clinical effectiveness study. Curr Med Res Opin 2010;26:1607-19.

28 Tamburin S, Schweiger V, Magrinelli F, et al. Effect of $5 \%$ lidocaine medicated plaster on pain intensity and paroxysms in classical trigeminal neuralgia. Ann Pharmacother 2014;48:1521-4.

29 Moore A, Derry S, Eccleston C, et al. Expect analgesic failure; pursue analgesic success. BMJ 2013;346:f2690.

30 McQuay HJ, Derry S, Moore AR, et al. Enriched enrolment with randomised withdrawal (EERW): time for a new look at clinical trial design in chronic pain. Pain 2008;135:217-20.

31 Toth C, Mawani S, Brady S, et al. An enriched-enrolment, randomized withdrawal, flexible-dose, double-blind, placebocontrolled, parallel assignment efficacy study of nabilone as adjuvant in the treatment of diabetic peripheral neuropathic pain. Pain 2012;153:2073-82

32 Huffman CL, Goldenberg JN, Weintraub J, et al. Efficacy and safety of once-daily controlled-release pregabalin for the treatment of patients with postherpetic neuralgia: a double-blind, enriched enrollment randomized withdrawal, placebo-controlled trial. Clin J Pain 2017;33:569-78.

33 Zakrzewska JM, Palmer J, Morisset V, et al. Safety and efficacy of a Nav1.7 selective sodium channel blocker in patients with trigeminal neuralgia: a double-blind, placebo-controlled, randomised withdrawal phase 2A trial. Lancet Neurol 2017;16:291-300.

34 Moore RA, Edwards JE, McQuay HJ. Acute pain: individual patient meta-analysis shows the impact of different ways of analysing and presenting results. Pain 2005;116:322-31.

35 Cleeland CS, Ryan KM. Pain assessment: global use of the brief pain inventory. Ann Acad Med Singap 1994;23:129-38.

36 Buysse DJ, Reynolds CF, Monk TH, et al. The pittsburgh sleep quality index: a new instrument for psychiatric practice and research. Psychiatry Res 1989;28:193-213.

37 Brazier JE, Harper R, Jones NM, et al. Validating the SF-36 health survey questionnaire: new outcome measure for primary care. BMJ 1992;305:160-4.

38 Martini A, Del Balzo G, Schweiger V, et al. Efficacy of lidocaine $5 \%$ medicated plaster (VERSATIS $R$ ) in patients with localized neuropathic pain poorly responsive to pharmacological therapy. Minerva Med 2018;109:344-51.

39 Chan A-W, Tetzlaff JM, Gøtzsche PC, et al. SPIRIT 2013 explanation and elaboration: guidance for protocols of clinical trials. BMJ 2013;346:e7586. 
40 Moher D, Hopewell S, Schulz KF, et al. CONSORT 2010 explanation and elaboration: updated guidelines for reporting parallel group randomised trials. BMJ 2010;340:c869.

41 Issue information-declaration of Helsinki. J Bone Miner Res 2019;34.

42 Campbell BJ, Rowbotham M, Davies PS, et al. Systemic absorption of topical lidocaine in normal volunteers, patients with post-herpetic neuralgia, and patients with acute herpes zoster. J Pharm Sci 2002:91:1343-50.

43 Campbell BJ, Rowbotham M, Davies PS, et al. Systemic absorption of topical lidocaine in normal volunteers, patients with post-herpetic neuralgia, and patients with acute herpes zoster. J Pharm Sci 2002;91:1343-50. 